\title{
Analysis of Gut Microbiota and Their Metabolic Potential in Patients with Schizophrenia Treated with Olanzapine: Results from a Six-Week Observational Prospective Cohort Study
}

\author{
Justyna Pełka-Wysiecka ${ }^{1}{ }^{\circledR}$, Mariusz Kaczmarczyk ${ }^{2}$, Agata Bąba-Kubiś ${ }^{1}$, Paweł Liśkiewicz ${ }^{1}($, \\ Michał Wroński ${ }^{1}$, Karolina Skonieczna-Żydecka ${ }^{3}{ }^{\circledR}$, Wojciech Marlicz $\left.{ }^{4}{ }^{(}\right)$, Błażej Misiak ${ }^{5}$, \\ Teresa Starzyńska ${ }^{4}$, Jolanta Kucharska-Mazur ${ }^{1}$, Igor Łoniewski ${ }^{1}$ * and Jerzy Samochowiec ${ }^{1}$ (D) \\ 1 Department of Psychiatry, Pomeranian Medical University in Szczecin, Broniewskiego 26, 71-460 Szczecin, \\ Poland; wysiecki@wp.pl (J.P.-W.); agata0621@gmail.com (A.B.-K.); pjliskiewicz@gmail.com (P.L.); \\ mwronski@pum.edu.pl (M.W.); jola_kucharska@tlen.pl (J.K.-M.); samoj@pum.edu.pl (J.S.) \\ 2 Department of Clinical and Molecular Biochemistry, Pomeranian Medical University in Szczecin, \\ Powstańców Wielkopolskich 72, 70-111 Szczecin, Poland; mariush@pum.edu.pl \\ 3 Department of Human Nutrition and Metabolomics, Pomeranian Medical University in Szczecin, \\ Broniewskiego 24, 71-460 Szczecin, Poland; karzyd@pum.edu.pl \\ 4 Department of Gastroenterology, Pomeranian Medical University in Szczecin, Unii Lubelskiej 1, \\ 71-252 Szczecin, Poland; marlicz@hotmail.com (W.M.); testa@pum.edu.pl (T.S.) \\ 5 Department of Genetics, Wroclaw Medical University, Marcinkowskiego 1, 50-368 Wrocław, Poland; \\ mblazej@interia.eu \\ * Correspondence: sanprobi@sanprobi.pl; Tel.: +48-91-441-4806
}

Received: 13 August 2019; Accepted: 25 September 2019; Published: 3 October 2019

\begin{abstract}
Accumulating evidence indicates the potential effect of microbiota on the pathogenesis and course of schizophrenia. However, the effects of olanzapine, second-generation antipsychotics, on gut microbiota have not been investigated in humans. This study aimed to analyze fecal microbiota in schizophrenia patients treated with olanzapine during six weeks of their hospital stay. After a seven-day washout from all psychotropic medications, microbiota compositions were evaluated at baseline and after six weeks of hospitalization using $16 \mathrm{~S}$ rRNA sequencing. The study was conducted in 20 inpatients, who followed the same hospital routine and received 5-20 mg daily doses of olanzapine. Olanzapine treatment was associated with clinical improvements in all patients and significant increases in body mass index in females, but not changes in gut microbiota compositions and predicted function. The severity of symptoms at the beginning of treatment varied in accordance with the predicted metabolic activity of the bacteria. The present findings indicate that the microbiota of schizophrenia patients is highly individual and has different taxonomical (Type 1, with a predominance of Prevotella, and Type 2 with a higher abundance of Bacteroides, Blautia and Clostridium) and functional clusters, and it does not change following six weeks of olanzapine therapy; in addition, the microbiota is not associated with either the weight gain observed in women or the effectiveness of olanzapine therapy.
\end{abstract}

Keywords: microbiota; schizophrenia; olanzapine administration; weight gain

\section{Introduction}

More than 21 million people worldwide suffer from schizophrenia (SZ) [1]. A growing body of studies has shown the role of the gut-brain axis dysregulation in the pathophysiology of SZ. Subclinical inflammation, aberrant monoamine metabolism, and abnormal hypothalamic-pituitary-adrenal axis 
activation have been widely reported in patients with SZ [2-5] and are associated with microbiota alterations [6-9]. For instance, Schwartz et al. [10] found elevated abundance of Lactobacillaceae, Halothiobacillaceae, Brucellaceae, and Micrococcineae and lowered counts of Veillonellaceae in a cohort of SZ patients; in addition, greater microbial abnormalities, lower remission rates, and poorer responses to therapy, as well as decreased microbiome $\alpha$-diversity index and altered gut microbial composition, were observed in SZ patients [11]. Although mechanisms underlying the potential effect of microbiota on the pathogenesis and course of SZ are yet to be determined, chronic inflammation [12] and altered tryptophan metabolism $[13,14]$ have been suggested to be implicated in the pathogenesis of SZ. However, gut microbiota-associated biomarkers that would hold clinical utility have not been indicated to date.

Olanzapine (OLZ), one of the most widely used second-generation antipsychotics (SGAs) [15], has multiple adverse effects, including weight gain, dyslipidemia, impaired glucose metabolism, and hypertension [16-19]. These metabolic adversities may occur shortly after treatment implementation and progress with treatment duration [20-22]. Importantly, the first year of antipsychotic treatment is a critical period for weight gain and other metabolic adverse effects [23]. Notably, weight gain at the beginning of OLZ therapy can be used to predict long-term outcomes related to cardiovascular comorbidity. Therefore, dietary counseling and weight management, including regular bodyweight measurements, should be implemented as soon as the OLZ therapy begins [24,25]. However, weight gain is of multifactorial nature [20,26-28], and, to date, no effective therapeutic strategies could prevent weight gain in patients treated with OLZ.

A few studies have demonstrated that OLZ administration plays a role in weight gain and metabolic malfunctions. Davey et al. [29] found that OLZ treatment induced metabolic alterations via microbiota changes, and the metabolic alterations could be reversed by treatment with antibiotics; in addition, microbial, inflammatory, and metabolic adversities related to OLZ treatment were sex-dependent [30]. Moreover, Morgan et al. [31] observed that weight gain depended on gut microbiota, and specific bacteria were responsible for weight gain. Furthermore, Flowers et al. [32] revealed that clusters of gut microbiota were associated with pharmacological treatment in patients with bipolar disorder. However, to the best of our knowledge, the effects of OLZ on gut microbiota in patients with SZ have not been investigated. We hypothesized that short-term treatment with OLZ in controlled conditions (unified dietary intake and environmental factors) affects fecal microbiota compositions, and microbiota can affect body weight and treatment efficacy. Accordingly, this study analyzed microbiota compositions of stool samples collected from a cohort of SZ inpatients. The cohort comprised of acutely-relapsed SZ inpatients who were followed-up for six weeks during OLZ treatment.

\section{Materials and Methods}

\subsection{Patients}

The study protocol was approved by the Bioethics Committee of the Pomeranian Medical University in Szczecin (Poland). All participants received a written description of the study aims and provided written informed consent before participation. Participants were recruited as inpatients at the Department of Psychiatry in Szczecin (Poland) between October 2016 and May 2018, and only 20 psychiatric inpatients met the inclusion criteria. The flow chart of the study design is shown in Figure 1. SZ was diagnosed based on the ICD (International Statistical Classification of Diseases and Related Health Problems) -10 criteria. 


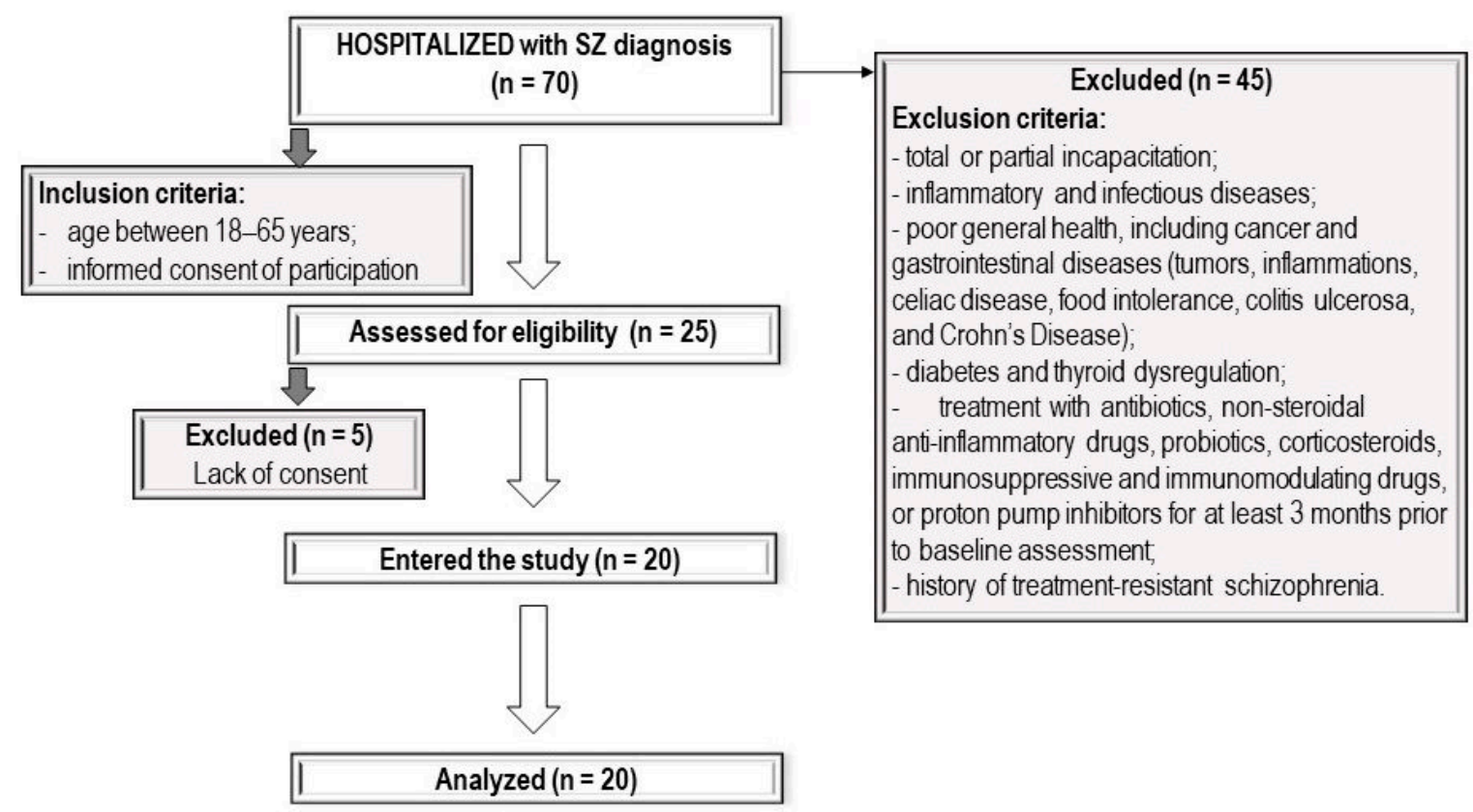

Figure 1. Flow chart of the study design. SZ, schizophrenia.

\subsection{Study Protocol}

All participants were subjected to the same daily activities, including physical exercise (daily morning exercise and a walk with a therapist), occupational therapy, and psycho-educational activities. Two senior psychiatrists performed the psychiatric and basic physical examinations, and a gastroenterologist conducted a comprehensive physical examination.

Patients received a standard hospital diet (i.e., $2995 \pm 93 \mathrm{kcal}, 106 \pm 14 \mathrm{~g}$ total protein, $420 \pm 24 \mathrm{~g}$ carbohydrates, and $102 \pm 10 \mathrm{~g}$ fat per day), balanced by a hospital dietician, in accordance with the Polish standards for hospitalized patients [33]. Detailed nutritional data on the diet during hospitalization, including fiber consumption, are presented in Supplementary Table S1.

This study included 20 patients, with 11 males and 9 females. After admission to the hospital ward, they were all subjected to a 7-day washout from psychiatric medications, received the standard hospital diet, and had a similar hospital routine. The first stool samples were collected after the washout period (W0), and subsequently, OLZ treatment was administered (initially $5 \mathrm{mg} /$ day; doses were individually adjusted up to $20 \mathrm{mg} /$ day). After 6 weeks of treatment, the second stool samples (W6) were collected (Supplementary Figure S1).

Clinical responses were defined as follows: Early responders, 30\% reduction in positive and negative syndrome scale (PANNS) total score at 4 weeks; late responders, $40 \%$ reduction in PANNS total score at end-point [34]; Clinical global impression-improvement scale (CGI-I) responders, score of 3 points (much improvement); and non-responders, clinical global impression-severity (CGI-S) scores of 4 (minimal improvement) or 5 (no improvement).

\subsection{Processing of Raw Data and Statistical Analysis}

Sequencing of the V4 region of $16 \mathrm{~S}$ rRNA gene was performed by the uBiome, Inc. (San Francisco, CA, USA). The $16 \mathrm{~S}$ amplicons from each sample were individually barcoded and sequenced in the multiplex in the NextSeq 500 platform in a $150 \mathrm{bp}$ (base pair) paired-end modality. The initial quality check of the $16 \mathrm{~S}$ sequences was conducted using the AfterQC (version 0.9.7) software with default settings [35]. Subsequently, forward and reverse reads were, respectively, capped at 125 and $124 \mathrm{bp}$ and then joined together with an in-between padding sequence ( 8 of "Ns" with a base score quality of 40 ). Each sequence was assigned the number of expected errors, and the sequences were filtered to have a maximum expected error of 1.0. The above steps were conducted using the VSEARCH (2.8.0) tool [36]. 
The sequences were processed using mothur (v.1.41.3) [37]. Briefly, sequences were aligned to the SILVA bacterial reference alignment (release 132), and were then screened to drop those not aligning to positions 13,148 and 25,277 of the SILVA alignment and were pre-clustered to allow two differences between sequences. The chimeras were identified and removed using VSEARCH implemented in mothur. Subsequently, sequences were classified using a Wang method with the Greengenes 16S rRNA Database version 13.8. Finally, sequences were clustered into OTUs using opticlust algorithm and Matthews correlation coefficient metric.

Metagenomic predictions from 16S rRNA marker genes (corrected for predicted 16S rRNA copy number) were carried out using PICRUSt (version 1.1.3) [38], and a list of the KEGG (Kyoto Encyclopedia of Genes and Genomes) functional orthologs (KO) was created. Reference genome coverage of the samples was calculated using the weighted nearest sequenced taxon index (NSTI) [38]. The PICRUSt predicted a median NSTI score of 0.11 (interquartile range, IQR of 0.05). The predicted metagenomes were analyzed with HUMAnN [39] and LEfSe [40]. The KO list was submitted as input data to HUMAnN, which generated KEGG modules and KEGG pathway abundances.

Downstream data analysis was performed using the R software (version 3.5.1, https://cran.rproject.org/), R based tools (such as Phyloseq package (version 1.24.2)) [41] and ComplexHeatmap [42], and custom-made scripts. Before calculating alpha diversity, the samples were rarefied to 3680 sequences per sample. Prior to beta diversity analysis, the taxa with the prevalence of less than $5 \%$ were removed (the prevalence of taxa was defined as the proportion of samples in which the taxa appeared at least once). Beta diversity was analyzed using principal coordinate analysis (PCoA) on Bray-Curtis distance matrices generated from the relative OTU abundances. To analyze the changes in bacterial community composition, a change in the principal coordinate 1 (PC1) was examined. The statistical analysis methods included the Wilcoxon rank-sum test, paired Wilcoxon signed-rank test, $t$-test for one sample, and Spearman rho correlation coefficient. $p$-values were adjusted using the Benjamini-Hochberg's false discovery rate (FDR) controlling procedure. Numerical data are presented as median, lower quartile, and upper quartile.

\section{Results}

\subsection{Microbiota Compositions}

General characteristics of patients are shown in Table 1. There was no significant change in alpha diversity as measured by Chao1 and Shannon indexes $(p=0.955$ and $p=0.808$, respectively; Figure 2A). The PCoA with Bray-Curtis dissimilarity is presented in Figure 2B. Samples were separated into distinct regions, mainly along the PC1 (Axis.1) that explained $42.5 \%$ of the intersample variance. The gut microbiome was individually specific, and the Bray-Curtis distances between the same samples were significantly smaller than those between all W0 samples ( $p=0.00006$; Figure $2 \mathrm{C})$. The direction of change along the PC1 was not consistent (Supplementary Figure S2). The mean change in the PC1 was not significantly different from 0 (0.0012, (95\% confidence interval: $-0.0946,0.0970), t=-0.03$, $\mathrm{df}=19, p=0.979)$, suggesting that the gut microbial community composition does not change after six weeks of treatment. In line with this observation, no OTUs were differentially abundant (from the genus to phylum level) between W0 and W6 (Supplementary Figures S3-S5). There was no change in the ratio of Firmicutes to Bacteroidetes $(\mathrm{F} / \mathrm{B})$ in the whole group, as well as in males and females (Supplementary Figure S6). In addition, there were no significant differences in the abundance of the KEGG orthologs, modules, and pathways between W0 and W6 samples in the whole group, as well as in men and women (Supplementary Figure S7). 
Table 1. Clinical characteristics of patients included in the study $(n=20)$.

\begin{tabular}{|c|c|}
\hline Variables & Median (1st Q-3rd Q)/ $n$ (\%) \\
\hline $\operatorname{Sex}(F / M)$ & $9(45 \%) / 11(55 \%)$ \\
\hline Age (years) & $33.5(31-39)$ \\
\hline $\operatorname{BMI}\left(\mathrm{kg} / \mathrm{m}^{2}\right)$ & $28.91(24.82-31.27)$ \\
\hline Olanzapine maximum dose (mg) & $20.00(20.0-20.0)$ \\
\hline Olanzapine average dose per day (mg) & $15.54(13.50-16.34)$ \\
\hline Disease duration (months) & $90(32-114)$ \\
\hline Duration of untreated psychosis (months) & $4.5(1.75-12.0)$ \\
\hline Smoking (number of cigarettes per day) ${ }^{a}$ & $1.5(1.0-3.0)$ \\
\hline Coffee (number of cups) & $2.0(0.0-3.0)$ \\
\hline Tea (number of cups) & $2.5(1.0-3.0)$ \\
\hline
\end{tabular}

BMI-Body Mass Index; ${ }^{\text {a }}$ Ordinal variables (per day): 1, non-smokers; 2 , up to 10 cigarettes; 3 , up to 20 cigarettes; 4 , up to 40 cigarettes; 1 st $Q$, first quartile; 3 rd $Q$, third quartile, BMI—body mass index.

A
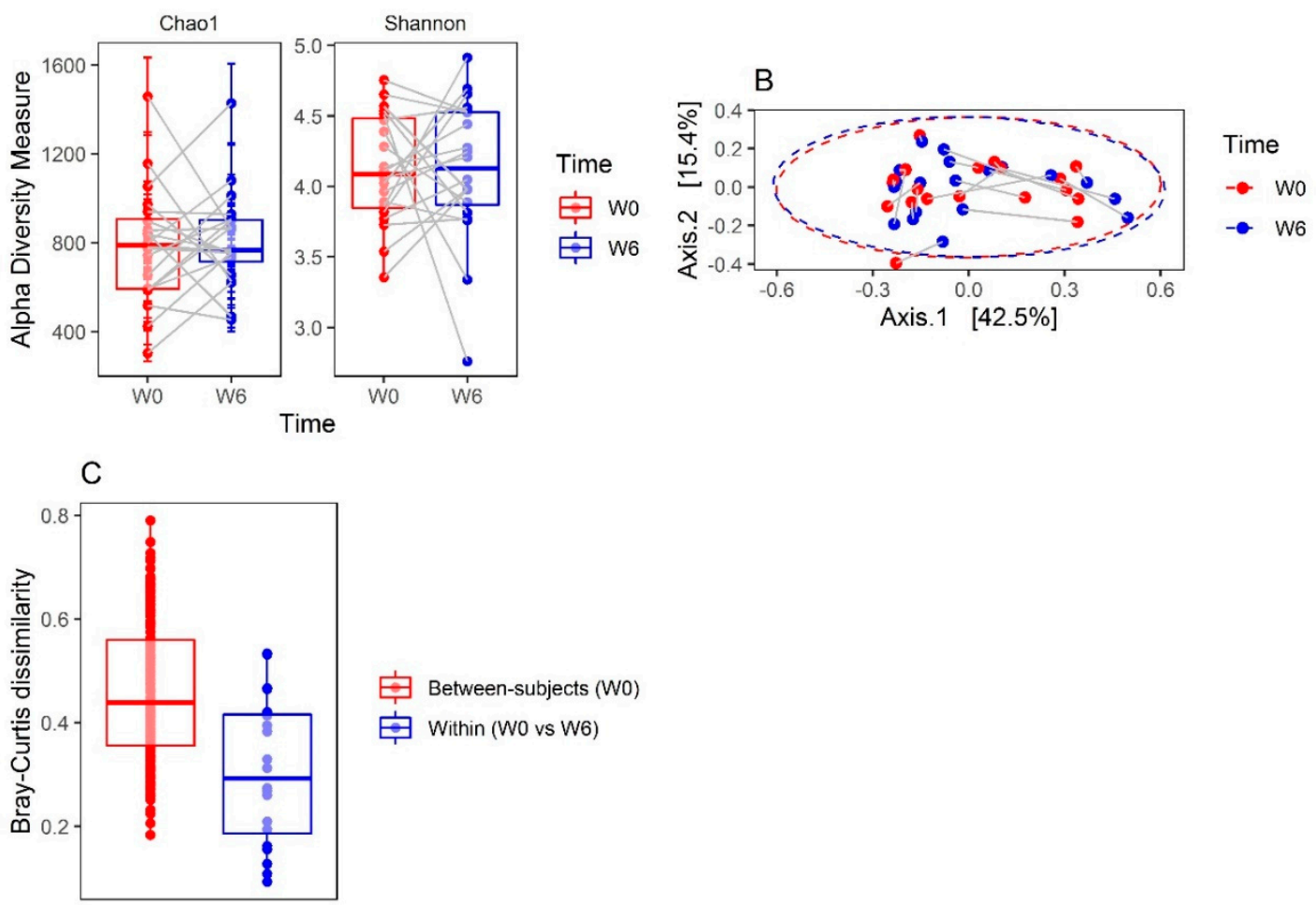

Figure 2. (A) Alpha diversity measures at baseline (W0) and after six weeks of hospitalization (W6). The boxplots represent the diversity measures (center line, median; lower and upper hinges correspond to the first (Q1) and third (Q3) quartiles; whiskers, 1.5 * IQR (Q3-Q1). Grey lines connect samples from the same patients. (B) Genus level resolution analysis of gut microbiota in patients diagnosed with paranoid schizophrenia treated with olanzapine during six weeks of hospitalization. The principal coordinate analysis was based on Bray-Curtis dissimilarities calculated using relative abundance data. Samples are colored according to time points (W0 and W6). Grey lines connect samples from the same patients. Ellipses correspond to 95\% confidence intervals for two timepoints (W0 and W6) with a multivariate normal distribution. (C) The boxplot shows Bray-Curtis dissimilarities calculated in the same patients (within (W0 vs. W6), 0.29 (0.19-0.42)) and in different patients (between subjects (W0), 0.44 (0.36-0.56), $p=0.00006$, Wilcoxon rank-sum test) (center line: median, lower, and upper hinges correspond to the first (Q1) and third (Q3) quartiles; whiskers: the upper whisker is located at the smaller of the maximum Bray-Curtis measures and Q3 + 1.5 * IQR (Q3-Q1); the lower whisker is located at the larger of the minimum Bray-Curtis measures and Q1-1.5 * IQR). W0 and W6 represent time points. 
Despite the lack of a consistent shift along the PC1, we examined whether the PC1 changes are associated with demographic, clinical, and environmental factors. The mean PC1 changes did not differ between men $(0.011(-0.118-0.140))$ and women $(0.004(-0.036-0.016))$ (Wilcoxon rank-sum test FDR adjusted $p(q)=0.649$ ). Demographic, clinical, and environmental factors were not correlated with the change in the PC1 (Supplementary Table S2, Supplementary Figure S8). There was no association between the dose of OLZ and the shift in the gut microbial composition (Supplementary Figure S9). However, the change in the PC1 was significantly greater in patients consuming alcohol (1-3 unit of alcohol; $0.16(0.03-0.32))$ than in non-alcohol drinkers $(-0.01(-0.19-0.01))$ (Wilcoxon rank-sum test $q=0.036$ ). To further explore the distinct regions revealed by the ordination of samples by PCoA (Figure 2B), we conducted an unsupervised hierarchical clustering using an average linkage algorithm of the Bray-Curtis dissimilarity. The clustering analysis showed the presence of two clusters (Type 1: 9 samples; Type 2: 31 samples) that matched with distinct regions revealed in the PCoA. A heatmap displaying the relative abundances for the genera annotated with two resulting clusters is shown in Figure 3A. Differential abundance testing revealed that the Bacteroides, Blautia, Clostridium, Anaerostipes, Bilophila, Anaerotruncus, and Eggerthella were enriched in the Type 2 cluster, whereas Prevotella was enriched in the Type1 cluster (Figure 3B). Clusters Type 1 and 2 seemed to correspond to enterotypes 2 (Prevotella) and 1 (Bacteroides) described by Arumugam et al. in 2011 [43], respectively. To explore these enterotypes in more detail, our cluster Type 2 was analyzed more thoroughly, as it seemed not to be completely homogenous. Cluster Type 2 was divided into Type 2A and Type 2B, and then the relative abundances of the main contributors from each enterotype in the resulting three clusters (Type 1 , Type 2A and 2B) were ascertained (Supplementary Figure S10) Cluster Type 1 had an abundance pattern similar to enterotype 2 (Figure $2 \mathrm{~d}$ in Arumugam et al. [43]), and that for cluster type 2A to enterotype 1 (Figure $2 \mathrm{~d}$ in Arumugam et al. [43]). However, cluster type 2B seemed not to be similar to any enterotype. Two genera (Prevotella and Bacteroides) exhibited similar abundance which was greater than of Ruminococcus. The pattern Type 2B seemed to be a type of a mixture from clusters Type 1 and Type 2A. The addition of Blautia made no difference to this assessment (Supplementary Figure S11).

A



B

\begin{tabular}{|c|c|c|c|}
\hline \multirow{2}{*}{ Genus } & \multicolumn{2}{|c|}{$\begin{array}{l}\text { Relative abundance (\%), median (lower } \\
\text { quartile - upper quartile) }\end{array}$} & \multirow{2}{*}{$\mathrm{q}^{\mathrm{a}}$} \\
\hline & Type1 (n=9) & Type2 (n=31) & \\
\hline Prevotella & $46(43-50)$ & $0.07(0.01-13.34)$ & 0.0008 \\
\hline Bacteroides & $7.9(6.3-11.8)$ & $27.1(16.8-32.7)$ & 0.001 \\
\hline Blautia & $2.5(1.8-4.1)$ & $6.5(4.1-9.4)$ & 0.024 \\
\hline Clostridium & $0.80(0.37-1.01)$ & $2.17(1.39-2.97)$ & 0.011 \\
\hline Anaerostipes & $0.09(0.01-1.03)$ & $0.17(0.11-0.23)$ & 0.041 \\
\hline Bilophila & $0.02(0.01-0.09)$ & $0.23(0.13-0.44)$ & 0.029 \\
\hline Anaerotruncus & $0.0009(0.0007-0.002)$ & $0.006(0.003-0.024)$ & 0.008 \\
\hline
\end{tabular}

Figure 3. (A) Genus level resolution analysis of gut microbiota in patients diagnosed with paranoid schizophrenia treated with olanzapine during six weeks of hospitalization. Unsupervised average linkage hierarchical clustering of gut microbiota at the genus level was conducted. Two resulting clusters (Type 1, blue and Type 2, red) are shown as the top annotation. Both samples (W0 and W6) of 15 patients were found in either Type 1 or Type 2 cluster (two patients in Type 1 and 13 patients in Type 2). Samples of the five patients (3SJP, 4SMA, 10SUG, 11SAS, and 12SMS) belonged to different clusters. (B) Differential abundance testing at the genus level between Type 1 and Type 2 clusters. ${ }^{a}$ two sided Wilcoxon signed-rank test, FDR adjusted $p$, the genera with the relative abundance $>1 \%$ in at least one cluster are shown, Eggerthella not shown due to low abundance. 
Taken together, our results suggest that the gut microbiota is highly individually specific, and the microbial community compositional changes during six weeks of OLZ treatment are not consistent across the patients.

\subsection{Clinical Improvement and BMI Changes}

We found that OLZ treatment was associated with significantly improved treatment efficacy as measured by PANNS, 36-item short form survey (SF36), and CGI-S scales (Supplementary Table S3). We further investigated whether these improvements are correlated with the change in microbiota compositions (as measured by a change in the PC1 component) and with demographic and clinical characteristics. No significant correlations were observed between clinical improvements and changes in microbiota composition (Supplementary Figure S12) or demographic and clinical characteristics, except the duration of untreated psychosis (DUP) (Supplementary Table S4).

In contrast to changes in the symptom severity of schizophrenia (Supplementary Table S3), there was no significant change in the patients' BMI during OLZ treatment $(q=0.763)$. However, the BMI change (W6 vs. W0 difference) was significantly higher in women than in men (Supplementary Figure S13) but did not correlate significantly with age, OLZ average dose per day, OLZ maximum dose, disease duration, or duration of untreated psychosis.

Because we found clear differences in gut microbiome compositions in all 40 samples (Figure 3), we next sought to determine whether similar differences in microbial community compositions and metabolic potentials exist in baseline samples and whether those differences could affect the patients' clinical improvement and change in BMI within six weeks. We performed the unsupervised average linkage hierarchical clustering of the Bray-Curtis dissimilarity among the baseline samples (W0, Supplementary Figure S14), as well as that of the relative abundances of the predicted KEGG orthologs, modules, and pathways (Supplementary Figures S15-S17). Regarding the microbiome compositions, we were able to demonstrate different groups of patients (clusters) using hierarchical clustering of KEGG features in the W0 samples: KEGG orthologs (Supplementary Figure S15), modules (Supplementary Figure S16), and pathways (Supplementary Figure S17). Differential abundance testing revealed that only the Prevotella genus differed between the two clusters (Type 1, $0.01 \%(0.006-0.004)$ vs. Type 2, 27.4\% (17.7-43.1); two-sided Wilcoxon signed-rank test, FDR adjusted $p=0.033$; Supplementary Figure S14). To identify differentially abundant genes, modules, and pathways between clusters, we conducted a linear discriminant analysis with effect size (LEfSe) method (Figure 4).

Subsequently, we compared the baseline symptom scales and BMI between Type 1 and Type 2 clusters. We found significant differences in the baseline PANNS, PANNS G, and CGI-S between the groups created from the clustering of the pathway abundance (Table 2). The patients classified into a Type 2 cluster had significantly more severe symptoms at baseline. The improvement in symptom severity after OLZ treatment assessed by PANNS, SF36, and CG1I was not associated with microbial community compositions (Supplementary Figure S14, Table S5) or KEGG features at baseline (Table 2; Supplementary Figures S15-S17 and Tables S6 and S7). Likewise, no associations were found between baseline gut microbiota (Supplementary Figure S14, Supplementary Table S5) or its metabolic potentials (Table 2 and Supplementary Figures S15-S17 and Supplementary Tables S6 and S7) and the BMI change in the whole group or separately in women or men. 
A

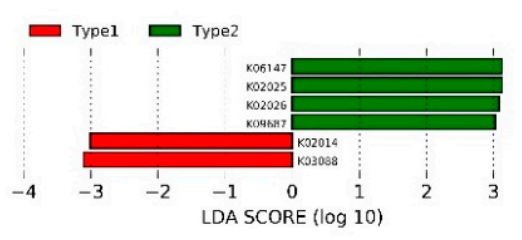

C



B

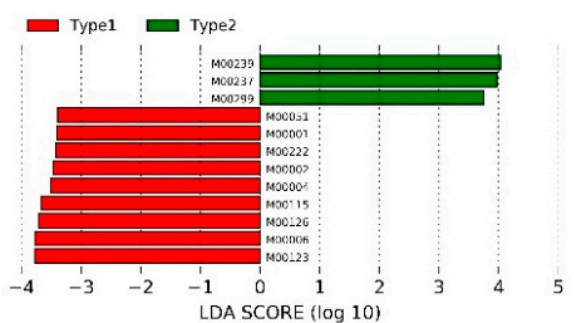

Figure 4. Unsupervised average linkage hierarchical clustering was carried out for each KEGG feature category. (A) KEGG orthologs; (B) KEGG modules; (C) KEGG pathways. K06147, ATP-binding cassette, subfamily B, bacterial; K02025, K02026, multiple sugar transport system permease proteins; K09687, antibiotic transport system ATP-binding protein; K02014, iron complex outer-membrane receptor protein; K03088, RNA polymerase sigma-70 factor, ECF subfamily; M00239, peptides/nickel transport system; M00237, branched-chain amino acid transport system; M00299, Spermidine/putrescine transport system; M00051, Uridine monophosphate biosynthesis, glutamine (+ PRPP) $\rightarrow$ UMP; M00222, phosphate transport system; M00002, glycolysis, core module involving three-carbon compounds; M00004, pentose phosphate pathway (pentose phosphate cycle); M00115, NAD biosynthesis, aspartate $\rightarrow$ NAD; M00126, Tetrahydrofolate biosynthesis, GTP $\rightarrow$ THF; M00006, pentose phosphate pathway, oxidative phase, glucose $6 \mathrm{P} \rightarrow$ ribulose 5P; M00123, Biotin biosynthesis, pimeloyl-CoA $\rightarrow$ biotin; ko00511, other glycan degradation; ko00531, glycosaminoglycan degradation; ko00540, lipopolysaccharide biosynthesis; ko00780, biotin metabolism; ko00790, folate biosynthesis; ko00130, ubiquinone and other terpenoid-quinone biosynthesis; ko00460, cyanoamino acid metabolism; ko00020, citrate cycle (TCA cycle); ko00600, sphingolipid metabolism; ko00940, phenylpropanoid biosynthesis; ko00908, zeatin biosynthesis; ko00250, alanine, aspartate and glutamate metabolism; ko00430, taurine and hypotaurine metabolism; ko00040, pentose and glucuronate interconversions; ko00030, pentose phosphate pathway; ko02060, phosphotransferase system (PTS); ko00290, valine, leucine and isoleucine biosynthesis; ko00072, synthesis and degradation of ketone bodies; ko00860, porphyrin and chlorophyll metabolism; ko04122, sulfur relay system; ko02010, ABC transporters; ko00061, fatty acid biosynthesis; ko02030, bacterial chemotaxis. 
Table 2. Associations of KEGG pathways with BMI changes and clinical improvements (PANNS, SF36, and CGI).

\begin{tabular}{cccc}
\hline Variables (Females + Males) & Cluster Type 2 $(n=5)$ & Cluster Type 3 $(n=12)$ & $p / q^{\mathbf{a}}$ \\
\hline BMI (kg/m $\left.{ }^{2}\right)$ W0 & $28.7(27-29.9)$ & $29.6(24.4-32)$ & $0.874 / 0.874$ \\
PANNS W0 & $95(94-98)$ & $68(62.8-74.2)$ & $0.007 / 0.047$ \\
PANNS N subscale W0 & $28(23-28)$ & $20.5(17-22.2)$ & $0.020 / 0.070$ \\
PANNS P subscale W0 & $24(23-26)$ & $20(15.8-22)$ & $0.026 / 0.073$ \\
PANNS G subscale W0 & $46(43-47)$ & $32(27.8-37)$ & $0.010 / 0.047$ \\
SF36 W0 & $90(83-97)$ & $76.5(72.5-83.8)$ & $0.102 / 0.238$ \\
CGI-S W0 & $7(6-7)$ & $5(5-6)$ & $0.009 / 0.047$ \\
BMI (kg/m2) & $-0.53(-1.33-0.72)$ & $0.35(-0.23-0.90)$ & $0.562 / 0.656$ \\
PANNS & $-44(-65--31)$ & $-37(-39.5--21.8)$ & $0.342 / 0.749$ \\
PANNS N subscale & $-10(-17--9)$ & $-6(-8.75--4.5)$ & $0.205 / 0.410$ \\
PANNS P subscale & $-12(-18--6)$ & $-11(-15--7.75)$ & $0.874 / 0.874$ \\
PANNS G subscale & $-22(-26--16)$ & $-16(-17.8--8.75)$ & $0.315 / 0.479$ \\
SF36 & $-5(-18--4)$ & $-3(-12.2-6)$ & $0.245 / 0.429$ \\
CGI-I & $4(3-4)$ & $3.5(3-4)$ & $0.452 / 0.575$ \\
\hline Variable (Males) & Cluster Type 2 $(n=5)$ & Cluster Type 3 $(n=4)$ & $p / q^{\mathbf{a}}$ \\
\hline BMI (kg/m $\left.{ }^{2}\right)$ W0 & $28.7(27-29.9)$ & $30.5(27.5-32.2)$ & $0.713 / 0.768$ \\
PANNS W0 & $95(94-98)$ & $67(59.2-76.5)$ & $0.037 / 0.198$ \\
PANNS N subscale W0 & $28(23-28)$ & $20(18.8-22.2)$ & $0.084 / 0.198$ \\
PANNS P subscale W0 & $24(23-26)$ & $17(14-19)$ & $0.027 / 0.198$ \\
PANNS G subscale W0 & $46(43-47)$ & $30.5(24.8-37.5)$ & $0.065 / 0.198$ \\
SF36 W0 & $90(83-97)$ & $83.5(76.2-90)$ & $0.391 / 0.547$ \\
CGI-S W0 & $7(6-7)$ & $5.5(5-6)$ & $0.050 / 0.198$ \\
BMI (kg/m $\left.{ }^{2}\right)$ & $-0.53(-1.33-0.72)$ & $-0.92(-1.97--0.30)$ & $0.713 / 0.768$ \\
PANNS & $-44(-65--31)$ & $-20.5(-26.2--17.8)$ & $0.140 / 0.280$ \\
PANNS N subscale & $-10(-17--9)$ & $-6(-7.5--5.75)$ & $0.389 / 0.547$ \\
PANNS P subscale & $-12(-18--6)$ & $-7.5(-8.25--6)$ & $0.389 / 0.547$ \\
PANNS G subscale & $-22(-26--16)$ & $-8.5(-11--7)$ & $0.085 / 0.198$ \\
SF36 & $-5(-18--4)$ & $-11(-13.5--7)$ & $1.0 / 1.0$ \\
CGI-I & $4(3-4)$ & $4(4-4)$ & $0.661 / 0.768$ \\
\hline
\end{tabular}

a Two-sided Wilcoxon rank-sum test, median with lower and upper quartiles in parentheses; BMI, PANNS, and SF36-changes from baseline (W0); CGI-I—an improvement from baseline; KEGG, Kyoto Encyclopedia of Genes and Genomes; BMI, body mass index; PANNS, positive and negative syndrome scale; SF36, 36- item short form survey; CGI, clinical global impression-improvement scale.

To further explore the gut microbiota and OLZ treatment interactions, we classified the included patients as responders and non-responders as follows: Early responders, early non-responders, late responders, and late non-responders using the PANNS total score and responders and non-responders using the CGI-I scale. Subsequently, microbial community compositions and KEGG features were compared between responders and non-responders. Phylogenetic compositions of the samples at the phylum level in the responders and non-responders are shown in Supplementary Figure S18. The phyla were not differentially abundant in responders and non-responders, regardless of the definition of clinical improvement. There were no differences in gut microbiome compositions at other taxonomic levels (Supplementary Figure S19), as well as in the KEGG orthologs, modules, and pathways (Supplementary Figure S20). Sex-specific results are shown in Supplementary Figures S21 and S22 (bacterial community composition) and S23 and S24 (KEGG features).

\section{Discussion}

The effect of OLZ on the microbiota has been investigated in experimental studies. Davey et al. [29,30] found decreased gut microbiota diversity, increased abundance of phyla Firmicutes, and reduced Actinobacteria, Proteobacteria, and Bacteroidetes in the course of OLZ treatment in female rats. Similarly, Morgan et al. [31] revealed decreased alpha diversity, lower abundance of class 
Bacteroidia, and increased abundances of Erysipelotrichia, Actinobacteria, and Gammaproteobacteria in female mice treated with OLZ. However, Kao et al. [44] demonstrated no significant effects of OLZ on gut microbiota in female rats. To the best of our knowledge, this study is the first to analyze fecal microbiota compositions in patients hospitalized due to acute relapse of SZ. We did not find the impact of six-week OLZ treatment on bacterial diversity, abundance, and predicted metabolic function, and patients with SZ had individualized and stable gut microbiota in the course of six-week OLZ treatment in terms of both composition and function. Because of the inconsistent findings above, further studies are needed to clarify the effect of OLZ on gut microbiota.

Although gut microbiota could be compositionally and functionally clustered into similar groups, the classification could not be used to predict the responses to OLZ treatment or the occurrence of weight gain (observed only in women) during OLZ treatment. As mentioned above, OLZ causes weight gain in female rats [29,44] and mice [31]. This metabolic effect is not observed during antibiotic therapy [29] and gnotobiosis (germ-free mouse model) and is enhanced during the administration of the high-fat diet regimen that is responsible for alterations of microbiota similar to those observed in metabolic syndromes [31]. In addition, Davey et al. [30] demonstrated metabolic disturbances, inflammation, and microbiota alterations in female mice treated with OLZ and found only slight alterations in male mice treated with OLZ, and metabolic effects of OLZ were linked to gut microbiota alterations. Notably, antibiotics reversed these effects due to reduced gut microbiota. Therefore, changed gut microbiota plays a pivotal role in weight gain. The lack of association between fecal microbiota compositions and weight gain in this study may be due to the low number of participants and the short period of OLZ administration. In addition, other factors might also be responsible for the increase in body mass index associated with the OLZ administration [20,26-28].

In the present study two bacterial enterotypes (clusters) were found, Type 1, with a predominance of Prevotella, and Type 2 with a higher abundance of Bacteroides, Blautia and Clostridium. Cluster Type 2 seemed not to be completely homogenous (with Types 2A and 2B), which initially suggested the possibility of the occurrence of a third enterotype similar to that found by Arumugam et al. [43]. Further analysis did not confirm this hypothesis and a higher abundance of Ruminococcus or Blautia in sub-cluster Type 2B was not seen. This sub-cluster seemed to be a type of mixture from clusters Type 1 and Type 2A. Due to this we took into consideration in further analyses only two enterotypes (original clusters) of bacteria. Moreover, patients with SZ were clustered at the level of KEGG genes, modules, and pathways. The severity of symptoms measured at the beginning of treatment varied, depending on the predicted metabolic activity of the bacteria. Other studies also have observed a relationship between the composition of bacteria and the severity of symptoms in SZ patients. Zheng et al. [11] demonstrated that PANSS was negatively correlated with Veillonellaceae and was positively correlated with Bacteroidaceae, Streptococcaceae, and Lachnospiraceae. Schwartz et al. [10] found greater microbial abnormalities in SZ patients than in controls. In addition, increases in the number of Lactobacillus group bacteria were positively correlated with the severity of various symptom domains in SZ patients and were negatively correlated with the global assessment of functioning. Moreover, responses to the treatment were worse in patients with severe microbiota alterations. Furthermore, Shen et al. [45], using the PICRUSt analysis, infer that vitamin B6 and fatty acid metabolic potential differed significantly between SZ patients and controls. Therefore, there are potential relationships between predicted metabolic changes and the severity of symptoms in SZ patients, as shown in Table 3. It is important to note that the PICRUSt approach using in prediction of bacterial metabolic activity should be treated with caution and followed by metagenomic analyses to explain such findings in humans. The median NSTI score was 0.11 (interquartile range of 0.05 ) suggesting a reasonable accuracy of the prediction, however, some closely related reference genomes were not available. 
Table 3. Potential relationships between predicted metabolic changes and the severity of symptoms in schizophrenia (SZ) patients.

\begin{tabular}{|c|c|c|c|}
\hline Pathways & Physiological Function & Potential Roles in SZ & References \\
\hline \multicolumn{4}{|c|}{ Pathways found to be more active in patients with significantly less severe symptoms (according to PANNS and CGI-S) } \\
\hline $\begin{array}{l}\text { ko00430: Taurine and hypotaurine } \\
\text { metabolism }\end{array}$ & $\begin{array}{l}\text { Taurine: N-methyl-D-aspartate (NDMA) receptor inhibition } \\
\text { and stem cell activation; a neurotransmitter and an } \\
\text { inhibitory neuromodulator in the central nervous system } \\
\text { (CNS); a potential immunomodulating compound, and an } \\
\text { attenuator of apoptosis }\end{array}$ & $\begin{array}{l}\text { Taurine supplementation was found to alleviate SZ } \\
\text { symptoms significantly }\end{array}$ & [46-48] \\
\hline $\begin{array}{l}\text { Ko00250: Alanine (ALA), aspartate } \\
\text { (ASP), and glutamate metabolism }\end{array}$ & $\begin{array}{l}\text { ALA: An agonist that binds to the glycine site of NMDA } \\
\text { receptors and improves the positive and cognitive } \\
\text { symptoms of patients with SZ; ASP: Binding to the agonist } \\
\text { site of NMDARs }\end{array}$ & NMDAR hypofunction in schizophrenia pathogenesis & {$[49,50]$} \\
\hline Ko00790: Folate biosynthesis & Folate: Production of adenosylmethionine (SAM) & $\begin{array}{l}\text { Schizophrenia patients may have lower folate levels } \\
\text { (negative correlation with negative symptoms of SZ) }\end{array}$ & [51-53] \\
\hline $\begin{array}{l}\text { Ko00130: Ubiquinone and other } \\
\text { terpenoids-quinone biosynthesis }\end{array}$ & $\begin{array}{l}\text { Ubiquinone: ATP production, mitochondrial function, } \\
\text { and reduction of proinflammatory mediators }\end{array}$ & Mitochondrial dysfunction as a part of SZ etiology & {$[54,55]$} \\
\hline Ko00020: Citrate cycle (TCA cycle) & TCA: Normal energy metabolism of the brain & $\begin{array}{l}\text { Abnormalities in energy metabolism were found to play a } \\
\text { role in SZ pathophysiology }\end{array}$ & [56] \\
\hline $\begin{array}{l}\text { Ko00600: Sphingolipid (SL) } \\
\text { metabolism }\end{array}$ & $\begin{array}{l}\text { Formation of membrane "lipid rafts" of myelin sheaths, } \\
\text { especially in neurons and oligodendrocytes (crucial for } \\
\text { normal synaptic neurotransmission, axon-myelin stability, } \\
\text { and communication/connectivity) }\end{array}$ & $\begin{array}{l}\text { Inflammatory, synaptic, and white matter changes that } \\
\text { result in disconnectivity in SZ may be related to SL }\end{array}$ & {$[57,58]$} \\
\hline \multicolumn{4}{|c|}{ Pathways found to be more active in patients with significantly more severe symptoms (according to PANNS and CGI-S) } \\
\hline Ko00030: Pentose phosphate pathway & $\begin{array}{l}\text { Formation of NADPH for biosynthetic processes, } \\
\text { cellular redox balance, and synthesis of ribose }\end{array}$ & $\begin{array}{l}\text { Pentose phosphate pathway-related molecules in } \\
\text { schizophrenia were found to be increased }\end{array}$ & [59] \\
\hline Ko00061: Fatty acid biosynthesis & $\begin{array}{l}\text { Component of membranes and } \\
\text { myelination process mediator }\end{array}$ & $\begin{array}{l}\text { Lipolysis and } \beta \text {-oxidation were found to be upregulated in } \\
\text { SZ, as a result of insufficient brain energy supply }\end{array}$ & {$[60,61]$} \\
\hline $\begin{array}{l}\text { Ko00290: Valine, leucine, and } \\
\text { isoleucine biosynthesis }\end{array}$ & $\begin{array}{l}\text { Protein synthesis, production of energy, } \\
\text { compartmentalization of glutamate synthesis of amine } \\
\text { neurotransmitters, including serotonin, } \\
\text { dopamine, and norepinephrine }\end{array}$ & $\begin{array}{l}\text { Branched-chain amino acids when administered to patients } \\
\text { with tardive dyskinesia-aberration of voluntary motor } \\
\text { control in SZ patients treated with psychotropic drugs }\end{array}$ & {$[62,63]$} \\
\hline $\begin{array}{l}\text { Ko00072: Synthesis and degradation of } \\
\text { ketone bodies }\end{array}$ & $\begin{array}{l}\text { An alternative source of energy under fasting and starving; } \\
\text { restrictive diets prolonged intense exercise }\end{array}$ & $\begin{array}{c}\text { Ketones may change the ratio of GABA (glutamate in favor } \\
\text { of GABA) to compensate GABA levels in the } \\
\text { CNS in SZ patients }\end{array}$ & [64] \\
\hline
\end{tabular}

PANNS-The Positive and Negative Syndrome Scale, CGI-S-The Clinical Global Impressions Scale. 
Our study has several strengths that should be highlighted. (1) The applied treatment resulted in expected clinical effects. The relationship between duration of untreated psychosis (DUP) and poor general symptomatic outcomes was confirmed, and the longer DUP was associated with more severe positive and negative symptoms. Additionally, OLZ treatment caused weight gain. This observation is in agreement with that in another experimental study [65]. (2) During the treatment, the patients were under the same controlled hospital conditions (diet, drug intake, and clinical monitoring), and a washout was used before treatment, thus providing a "unification" of the environmental impact on the fecal microbiota pattern. Consequently, we speculate that such conditions diminish the impact of common environmental factors that permanently shape gut microbiota composition and underline the association between the disease and treatment. (3) Weight gain at the beginning of OLZ treatment is very important because it determines the further development of cardiometabolic risk factors $[22,23,66]$. (4) Although the study group was not homogeneous, the symptoms were observed every day in our psychiatric clinic. Patients were previously treated with other pharmaceuticals, which might have affected the microbiota composition. Such situations might lead to resistance against psychotropic drugs, probably leading to no impact of OLZ on the microbiota.

There are certain limitations of our study that need to be discussed. First, the sample size was small and heterogeneous (drug-naive and previously-treated patients). No formal sample size calculations were employed for this analysis, but the cohort size was based on what was previously sufficient to test microbiotic changes in schizophrenia patients [67], and/or the influence of antibiotics [68] and risperidone administration [69] on gut microbiota. This limitation should be attributed to rigorous inclusion and exclusion criteria as well as the short duration of the whole study (17 months). Thus, studies with a greater sample size are needed to further examine the associations between OLZ treatment and gut microbiota structure. Second, the composition of intestinal bacteria varied among individuals, and inter-individual variation within the gut ecosystem of patients was high. Third, in individual studies (also experimental), various taxonomic groups of bacteria were analyzed only in stools. The composition of bacteria in feces is more stable and is not influenced by external factors compared with the composition of bacteria in the small intestine. Changes in the microbiota of the small bowel have a much greater effect on the metabolic functions of the human body. Therefore, further experimental studies should pay more attention to this issue [70,71], although an invasive way of sampling intestine biological material remains difficult and holds several ethical concerns. Fourth, there was a lack of long-term follow-up, which is especially important in case of metabolic consequences of OLZ treatment. Fifth, we did not compare the results between SZ patients and healthy subjects or patients receiving placebos. Matched controls with similar lifestyle should be used to exclude false-positive results. However, the general lifestyle in patients diagnosed with SZ was found to be divergent from that observed in healthy people [72]. Therefore, a placebo approach was impossible mainly due to ethical and organizational concerns. Sixth, changes in dietary and living conditions during the hospital stay might be another limitation of our study. However, enterotypes Prevotella and Bacteroides are strongly associated with long-term diet. It was shown that microbiome composition changed detectably within $24 \mathrm{~h}$ of initiating a high-fat/low-fiber or low-fat/high-fiber diet, but that enterotype identity remained stable during the 10-day study [73]. Therefore, a change of diet after admission to hospital should not affect W0 microbiota. After this all patients received the same diet, and it seems that this factor should also not significantly affect the influence of OLZ on W6 microbiota composition.

\section{Conclusions}

In conclusion, the present findings indicate that the microbiota in patients with the schizophrenia episode is highly individualized, although it can be clustered into different taxonomical (Type 1, with a predominance of Prevotella, and Type 2 with a higher abundance of Bacteroides, Blautia, and Clostridium) and functional groups; the microbiota does not change during six weeks of treatment with OLZ and is not associated with the weight gain that occurs in women treated with OLZ, as well as the treatment 
effectiveness. This study provides some insights into the metabolic effects of psychotropic drugs on gut microbiota in SZ patients. Further long-term and placebo-controlled studies are needed to clarify the effect of OLZ on gut microbiota.

Supplementary Materials: The following are available online at http://www.mdpi.com/2077-0383/8/10/1605/s1.

Author Contributions: Conceptualization, J.P.-W., A.B.-K. and J.S.; data curation, J.P.-W., M.K., A.B.-K., P.L., M.W. and I.Ł.; formal analysis, J.P.-W., M.K., P.L., K.S.-Ż., W.M., B.M., J.K.-M., I.Ł. and J.S.; investigation, J.P.-W., M.K., A.B.-K., P.L., M.W., K.S.-Ż., W.M., J.K.-M., I.Ł. and J.S.; methodology, J.S.; software, M.K.; supervision, J.P.-W. and J.S.; visualization, M.K.; writing—original draft, J.P.-W., M.K. and I.Ł.; writing—review and editing, J.P.-W., M.K., A.B.-K., M.W., K.S.-Ż., W.M., B.M., T.S., I.Ł. and J.S.

Funding: The study was funded within the framework of the project Fundusz Stymulacji Nauki (grant number: FSN-312-05/15) provided by Pomeranian Medical University at Szczecin (Poland) and National Science Centre (UMO-2018/31/B/NZ5/00527). The funding sources had no role in concept design, selection of articles, the decision to publish, or the preparation of the manuscript.

Acknowledgments: We would like to thank Editage (www.editage.com) for English language editing.

Conflicts of Interest: I.Ł. and W.M. are cofounders and shareholders at Sanprobi company. However, the content of this study was not constrained by this fact. Other authors declare no conflicts of interest.

\section{References}

1. WHO Schizophrenia. Available online: https://www.who.int/news-room/fact-sheets/detail/schizophrenia (accessed on 2 June 2019).

2. Müller, N. Inflammation in Schizophrenia: Pathogenetic Aspects and Therapeutic Considerations. Schizophr. Bull. 2018, 44, 973-982. [CrossRef] [PubMed]

3. Purves-Tyson, T.D.; Owens, S.J.; Rothmond, D.A.; Halliday, G.M.; Double, K.L.; Stevens, J.; McCrossin, T.; Weickert, C.S. Putative presynaptic dopamine dysregulation in schizophrenia is supported by molecular evidence from post-mortem human midbrain. Transl. Psychiatry 2017, 7, e1003. [CrossRef] [PubMed]

4. Guest, F.L.; Martins-de-Souza, D.; Rahmoune, H.; Bahn, S.; Guest, P.C. The effects of stress on hypothalamic-pituitary-adrenal (HPA) axis function in subjects with schizophrenia. Arch. Clin. Psychiatry (São Paulo) 2013, 40, 20-27. [CrossRef]

5. Guest, P.C.; Martins-De-Souza, D.; Vanattou-Saifoudine, N.; Harris, L.W.; Bahn, S. Abnormalities in Metabolism and Hypothalamic-Pituitary-Adrenal Axis Function in Schizophrenia. Int. Rev. Neurobiol. 2011, 101, 145-168.

6. Clemente, J.C.; Manasson, J.; Scher, J.U. The role of the gut microbiome in systemic inflammatory disease. BMJ 2018, 360, j5145. [CrossRef]

7. Sudo, N.; Chida, Y.; Aiba, Y.; Sonoda, J.; Oyama, N.; Yu, X.-N.; Kubo, C.; Koga, Y. Postnatal microbial colonization programs the hypothalamic-pituitary-adrenal system for stress response in mice. J. Physiol. 2004, 558, 263-275. [CrossRef]

8. Waclawiková, B.; El Aidy, S. Role of Microbiota and Tryptophan Metabolites in the Remote Effect of Intestinal Inflammation on Brain and Depression. Pharmaceuticals (Basel) 2018, 11, 63. [CrossRef]

9. Rogers, G.B.; Keating, D.J.; Young, R.L.; Wong, M.-L.; Licinio, J.; Wesselingh, S. From gut dysbiosis to altered brain function and mental illness: Mechanisms and pathways. Mol. Psychiatry 2016, 21, 738-748. [CrossRef]

10. Schwarz, E.; Maukonen, J.; Hyytiäinen, T.; Kieseppä, T.; Orešič, M.; Sabunciyan, S.; Mantere, O.; Saarela, M.; Yolken, R.; Suvisaari, J. Analysis of microbiota in first episode psychosis identifies preliminary associations with symptom severity and treatment response. Schizophr. Res. 2018, 192, 398-403. [CrossRef]

11. Zheng, P.; Zeng, B.; Liu, M.; Chen, J.; Pan, J.; Han, Y.; Liu, Y.; Cheng, K.; Zhou, C.; Wang, H.; et al. The gut microbiome from patients with schizophrenia modulates the glutamate-glutamine-GABA cycle and schizophrenia-relevant behaviors in mice. Sci. Adv. 2019, 5, eaau8317. [CrossRef]

12. Rakoff-Nahoum, S.; Paglino, J.; Eslami-Varzaneh, F.; Edberg, S.; Medzhitov, R. Recognition of Commensal Microflora by Toll-Like Receptors Is Required for Intestinal Homeostasis. Cell 2004, 118, 229-241. [CrossRef] [PubMed]

13. Barry, S.; Clarke, G.; Scully, P.; Dinan, T.G. Kynurenine pathway in psychosis: Evidence of increased tryptophan degradation. J. Psychopharmacol. 2009, 23, 287-294. [CrossRef] [PubMed] 
14. Miller, C.L.; Llenos, I.C.; Dulay, J.R.; Weis, S. Upregulation of the initiating step of the kynurenine pathway in postmortem anterior cingulate cortex from individuals with schizophrenia and bipolar disorder. Brain Res. 2006, 1073, 25-37. [CrossRef] [PubMed]

15. Hálfdánarson, Ó.; Zoëga, H.; Aagaard, L.; Bernardo, M.; Brandt, L.; Fusté, A.C.; Furu, K.; Garuoliené, K.; Hoffmann, F.; Huybrechts, K.F.; et al. International trends in antipsychotic use: A study in 16 countries, 2005-2014. Eur. Neuropsychopharmacol. 2017, 27, 1064-1076.

16. Vancampfort, D.; Correll, C.U.; Galling, B.; Probst, M.; De Hert, M.; Ward, P.B.; Rosenbaum, S.; Gaughran, F.; Lally, J.; Stubbs, B. Diabetes mellitus in people with schizophrenia, bipolar disorder and major depressive disorder: A systematic review and large scale meta-analysis. World Psychiatry 2016, 15, 166-174. [CrossRef]

17. Galling, B.; Roldán, A.; Nielsen, R.E.; Nielsen, J.; Gerhard, T.; Carbon, M.; Stubbs, B.; Vancampfort, D.; De Hert, M.; Olfson, M.; et al. Type 2 Diabetes Mellitus in Youth Exposed to Antipsychotics. JAMA Psychiatry 2016, 73, 247-259. [CrossRef]

18. Galling, B.; Correll, C.U. Do antipsychotics increase diabetes risk in children and adolescents? Expert Opin. Drug Saf. 2015, 14, 219-241. [CrossRef]

19. De Hert, M.; Vancampfort, D.; Correll, C.U.; Mercken, V.; Peuskens, J.; Sweers, K.; Van Winkel, R.; Mitchell, A.J. Guidelines for screening and monitoring of cardiometabolic risk in schizophrenia: Systematic evaluation. Br. J. Psychiatry 2011, 199, 99-105. [CrossRef]

20. Bak, M.; Fransen, A.; Janssen, J.; Van Os, J.; Drukker, M. Almost All Antipsychotics Result in Weight Gain: A Meta-Analysis. PLoS ONE 2014, 9, e94112. [CrossRef]

21. Musil, R.; Obermeier, M.; Russ, P.; Hamerle, M. Weight gain and antipsychotics: A drug safety review. Expert Opin. Drug Saf. 2015, 14, 73-96. [CrossRef]

22. Spertus, J.; Horvitz-Lennon, M.; Abing, H.; Normand, S.-L. Risk of weight gain for specific antipsychotic drugs: A meta-analysis. NPJ Schizophr. 2018, 4, 12. [CrossRef] [PubMed]

23. Pérez-Iglesias, R.; Martínez-García, O.; Pardo-Garcia, G.; Amado, J.A.; Garcia-Unzueta, M.T.; Tabares-Seisdedos, R.; Crespo-Facorro, B. Course of weight gain and metabolic abnormalities in first treated episode of psychosis: The first year is a critical period for development of cardiovascular risk factors. Int. J. Neuropsychopharmacol. 2014, 17, 41-51. [CrossRef] [PubMed]

24. American Diabetes Association; American Psychiatric Association; American Association of Clinical Endocrinologists; North American Association for the Study of Obesity Consensus development conference on antipsychotic drugs and obesity and diabetes. Diabetes Care 2004, 27, 596-601.

25. De Hert, M.; Dekker, J.M.; Wood, D.; Kahl, K.G.; Holt, R.I.G.; Möller, H.-J. Cardiovascular disease and diabetes in people with severe mental illness position statement from the European Psychiatric Association (EPA), supported by the European Association for the Study of Diabetes (EASD) and the European Society of Cardiology (ESC). Eur. Psychiatry 2009, 24, 412-424. [CrossRef]

26. Albaugh, V.L.; Henry, C.R.; Bello, N.T.; Hajnal, A.; Lynch, S.L.; Halle, B.; Lynch, C.J. Hormonal and metabolic effects of olanzapine and clozapine related to body weight in rodents. Obesity (Silver Spring) 2006, 14, 36-51. [CrossRef]

27. Basson, B.R.; Kinon, B.J.; Taylor, C.C.; Szymanski, K.A.; Gilmore, J.A.; Tollefson, G.D. Factors Influencing Acute Weight Change in Patients with Schizophrenia Treated with Olanzapine, Haloperidol, or Risperidone. J. Clin. Psychiatry 2001, 62, 231-238. [CrossRef]

28. Ou, J.-J.; Xu, Y.; Chen, H.-H.; Fan, X.; Gao, K.; Wang, J.; Guo, X.-F.; Wu, R.-R.; Zhao, J.-P. Comparison of metabolic effects of ziprasidone versus olanzapine treatment in patients with first-episode schizophrenia. Psychopharmacology 2013, 225, 627-635. [CrossRef]

29. Davey, K.J.; Cotter, P.D.; O'Sullivan, O.; Crispie, F.; Dinan, T.G.; Cryan, J.F.; O'Mahony, S.M. Antipsychotics and the gut microbiome: Olanzapine-induced metabolic dysfunction is attenuated by antibiotic administration in the rat. Transl. Psychiatry 2013, 3, e309. [CrossRef]

30. Davey, K.J.; O’Mahony, S.M.; Schellekens, H.; O'Sullivan, O.; Bienenstock, J.; Cotter, P.D.; Dinan, T.G.; Cryan, J.F. Gender-dependent consequences of chronic olanzapine in the rat: Effects on body weight, inflammatory, metabolic and microbiota parameters. Psychopharmacology 2012, 221, 155-169. [CrossRef]

31. Morgan, A.P.; Crowley, J.J.; Nonneman, R.J.; Quackenbush, C.R.; Miller, C.N.; Ryan, A.K.; Bogue, M.A.; Paredes, S.H.; Yourstone, S.; Carroll, I.M.; et al. The Antipsychotic Olanzapine Interacts with the Gut Microbiome to Cause Weight Gain in Mouse. PLoS ONE 2014, 9, e115225. [CrossRef] 
32. Flowers, S.A.; Evans, S.J.; Ward, K.M.; McInnis, M.G.; Ellingrod, V.L. Interaction Between Atypical Antipsychotics and the Gut Microbiome in a Bipolar Disease Cohort. Pharmacother. J. Hum. Pharmacol. Drug Ther. 2017, 37, 261-267. [CrossRef] [PubMed]

33. Jarosz, M.; Rychlik, E.; Stoś, K.; Wierzejska, R.; Wojtasik, A.; Charzewska, J.; Mojska, H.; Szponar, L.; Sajór, I.; Kłosiewicz-Latoszek, L.; et al. Normy Żywienia dla Populacji Polski; Instytut Żywności i Żywienia: Warszawa, Poland, 2017; ISBN 978-83-86060-89-4.

34. Ascher-Svanum, H.; Zhao, F.; Detke, H.C.; Nyhuis, A.W.; Lawson, A.H.; Stauffer, V.L.; Montgomery, W.; Witte, M.M.; McDonnell, D.P. Early response predicts subsequent response to olanzapine long-acting injection in a randomized, double-blind clinical trial of treatment for schizophrenia. BMC Psychiatry 2011, 11, 152. [CrossRef] [PubMed]

35. Chen, S.; Huang, T.; Zhou, Y.; Han, Y.; Xu, M.; Gu, J. AfterQC: Automatic filtering, trimming, error removing and quality control for fastq data. BMC Bioinform. 2017, 18, 80. [CrossRef] [PubMed]

36. Rognes, T.; Flouri, T.; Nichols, B.; Quince, C.; Mahé, F. VSEARCH: A versatile open source tool for metagenomics. PeerJ 2016, 4, e2584. [CrossRef] [PubMed]

37. Schloss, P.D.; Westcott, S.L.; Ryabin, T.; Hall, J.R.; Hartmann, M.; Hollister, E.B.; Lesniewski, R.A.; Oakley, B.B.; Parks, D.H.; Robinson, C.J.; et al. Introducing mothur: Open-Source, Platform-Independent, Community-Supported Software for Describing and Comparing Microbial Communities. Appl. Environ. Microbiol. 2009, 75, 7537-7541. [CrossRef]

38. Langille, M.G.I.; Zaneveld, J.; Caporaso, J.G.; McDonald, D.; Knights, D.; Reyes, J.A.; Clemente, J.C.; Burkepile, D.E.; Thurber, R.L.V.; Knight, R.; et al. Predictive functional profiling of microbial communities using 16S rRNA marker gene sequences. Nat. Biotechnol. 2013, 31, 814-821. [CrossRef]

39. Abubucker, S.; Segata, N.; Goll, J.; Schubert, A.M.; Izard, J.; Cantarel, B.L.; Rodriguez-Mueller, B.; Zucker, J.; Thiagarajan, M.; Henrissat, B.; et al. Metabolic Reconstruction for Metagenomic Data and Its Application to the Human Microbiome. PLoS Comput. Biol. 2012, 8, e1002358. [CrossRef]

40. Segata, N.; Izard, J.; Waldron, L.; Gevers, D.; Miropolsky, L.; Garrett, W.S.; Huttenhower, C. Metagenomic biomarker discovery and explanation. Genome Biol. 2011, 12, R60. [CrossRef]

41. McMurdie, P.J.; Holmes, S. phyloseq: An R Package for Reproducible Interactive Analysis and Graphics of Microbiome Census Data. PLoS ONE 2013, 8, e61217. [CrossRef]

42. Gu, Z.; Eils, R.; Schlesner, M. Complex heatmaps reveal patterns and correlations in multidimensional genomic data. Bioinformatics 2016, 32, 2847-2849. [CrossRef]

43. Arumugam, M.; Raes, J.; Pelletier, E.; Le Paslier, D.; Yamada, T.; Mende, D.R.; Fernandes, G.R.; Tap, J.; Bruls, T.; Batto, J.-M.; et al. Enterotypes of the human gut microbiome. Nature 2011, 473, 174-180. [CrossRef] [PubMed]

44. Kao, A.C.-C.; Spitzer, S.; Anthony, D.C.; Lennox, B.; Burnet, P.W.J. Prebiotic attenuation of olanzapine-induced weight gain in rats: Analysis of central and peripheral biomarkers and gut microbiota. Transl. Psychiatry 2018, 8, 66. [CrossRef] [PubMed]

45. Shen, Y.; Xu, J.; Li, Z.; Huang, Y.; Yuan, Y.; Wang, J.; Zhang, M.; Hu, S.; Liang, Y. Analysis of gut microbiota diversity and auxiliary diagnosis as a biomarker in patients with schizophrenia: A cross-sectional study. Schizophr. Res. 2018, 197, 470-477. [CrossRef] [PubMed]

46. Firth, J.; Rosenbaum, S.; Ward, P.B.; Curtis, J.; Teasdale, S.B.; Yung, A.R.; Sarris, J. Adjunctive nutrients in first-episode psychosis: A systematic review of efficacy, tolerability and neurobiological mechanisms. Early Interv. Psychiatry 2018, 12, 774-783. [CrossRef] [PubMed]

47. Leppik, L.; Kriisa, K.; Koido, K.; Koch, K.; Kajalaid, K.; Haring, L.; Vasar, E.; Zilmer, M. Profiling of Amino Acids and Their Derivatives Biogenic Amines Before and After Antipsychotic Treatment in First-Episode Psychosis. Front. Psychol. 2018, 9, 155. [CrossRef] [PubMed]

48. O'Donnell, C.P.; Allott, K.A.; Murphy, B.P.; Yuen, H.P.; Proffitt, T.-M.; Papas, A.; Moral, J.; Pham, T.; O'Regan, M.K.; Phassouliotis, C.; et al. Adjunctive Taurine in First-Episode Psychosis: A Phase 2, Double-Blind, Randomized, Placebo-Controlled Study. J. Clin. Psychiatry 2016, 77, e1610-e1617. [CrossRef] [PubMed]

49. Hatano, T.; Ohnuma, T.; Sakai, Y.; Shibata, N.; Maeshima, H.; Hanzawa, R.; Suzuki, T.; Arai, H. Plasma alanine levels increase in patients with schizophrenia as their clinical symptoms improve-Results from the Juntendo University Schizophrenia Projects (JUSP). Psychiatry Res. Neuroimaging 2010, 177, 27-31. [CrossRef] 
50. Errico, F.; Nuzzo, T.; Carella, M.; Bertolino, A.; Usiello, A. The Emerging Role of Altered d-Aspartate Metabolism in Schizophrenia: New Insights from Preclinical Models and Human Studies. Front. Psychol. 2018, 9, 559. [CrossRef]

51. Cao, B.; Wang, D.-F.; Xu, M.-Y.; Liu, Y.-Q.; Yan, L.-L.; Wang, J.-Y.; Lu, Q.-B. Lower folate levels in schizophrenia: A meta-analysis. Psychiatry Res. 2016, 245, 1-7. [CrossRef]

52. Hussein, H.; El Mawella, S.A.; Ahmed, T. Folate, vitamin B12, and negative symptoms in schizophrenia. Egypt. J. Psychiatry 2018, 39, 89. [CrossRef]

53. Hill, M.; Shannahan, K.; Jasinski, S.; Macklin, E.A.; Raeke, L.; Roffman, J.L.; Goff, D.C. Folate supplementation in schizophrenia: A possible role for MTHFR genotype. Schizophr. Res. 2011, 127, 41-45. [CrossRef] [PubMed]

54. Maguire, Á.; Hargreaves, A.; Gill, M. Coenzyme Q10 and neuropsychiatric and neurological disorders: Relevance for schizophrenia. Nutr. Neurosci. 2018, 21, 1-14. [CrossRef] [PubMed]

55. Schmelzer, C.; Döring, F. Identification of LPS-inducible genes downregulated by ubiquinone in human THP-1 monocytes. BioFactors 2010, 36, 222-228. [CrossRef] [PubMed]

56. Bubber, P.; Hartounian, V.; Gibson, G.E.; Blass, J.P. Abnormalities in the tricarboxylic acid (TCA) cycle in the brains of schizophrenia patients. Eur. Neuropsychopharmacol. 2011, 21, 254-260. [CrossRef]

57. Castillo, R.I.; Rojo, L.E.; Henríquez-Henríquez, M.; Silva, H.; Maturana, A.; Villar, M.J.; Fuentes, M.; Gaspar, P.A. From Molecules to the Clinic: Linking Schizophrenia and Metabolic Syndrome through Sphingolipids Metabolism. Front. Mol. Neurosci. 2016, 10, 488. [CrossRef]

58. Weston-Green, K.; Babic, I.; De Santis, M.; Pan, B.; Montgomery, M.K.; Mitchell, T.; Huang, X.-F.; Nealon, J. Disrupted sphingolipid metabolism following acute clozapine and olanzapine administration. J. Biomed. Sci. 2018, 25, 40. [CrossRef]

59. Liu, M.-L.; Zhang, X.-T.; Du, X.-Y.; Fang, Z.; Liu, Z.; Xu, Y.; Zheng, P.; Xu, X.-J.; Cheng, P.-F.; Huang, T.; et al. Severe disturbance of glucose metabolism in peripheral blood mononuclear cells of schizophrenia patients: A targeted metabolomic study. J. Transl. Med. 2015, 13, 226. [CrossRef]

60. Yang, X.; Sun, L.; Zhao, A.; Hu, X.; Qing, Y.; Jiang, J.; Yang, C.; Xu, T.; Wang, P.; Liu, J.; et al. Serum fatty acid patterns in patients with schizophrenia: A targeted metabonomics study. Transl. Psychiatry 2017, 7, e1176. [CrossRef]

61. Peters, B.D.; Machielsen, M.W.J.; Hoen, W.P.; Caan, M.W.A.; Malhotra, A.K.; Szeszko, P.R.; Duran, M.; Olabarriaga, S.D.; de Haan, L. Polyunsaturated fatty acid concentration predicts myelin integrity in early-phase psychosis. Schizophr. Bull. 2013, 39, 830-838. [CrossRef]

62. Fernstrom, J.D. Branched-chain amino acids and brain function. J. Nutr. 2005, 135, 1539S-1546S. [CrossRef]

63. Bevans, M.L.; Read, L.L.; Chao, H.M.; Clelland, J.D.; Suckow, R.F.; Maher, T.J.; Citrome, L.; Richardson, M.A. Efficacy of the Branched-Chain Amino Acids in the Treatment of Tardive Dyskinesia in Men. Am. J. Psychiatry 2003, 160, 1117-1124.

64. Włodarczyk, A.; Wiglusz, M.S.; Cubała, W.J. Ketogenic diet for schizophrenia: Nutritional approach to antipsychotic treatment. Med. Hypotheses 2018, 118, 74-77. [CrossRef] [PubMed]

65. Penttilä, M.; Jaaskelainen, E.; Hirvonen, N.; Isohanni, M.; Miettunen, J. Duration of untreated psychosis as predictor of long-term outcome in schizophrenia: Systematic review and meta-analysis. Br. J. Psychiatry 2014, 205, 88-94. [CrossRef] [PubMed]

66. Lin, C.-H.; Lin, S.-C.; Huang, Y.-H.; Wang, F.-C.; Huang, C.-J. Early prediction of olanzapine-induced weight gain for schizophrenia patients. Psychiatry Res. 2018, 263, 207-211. [CrossRef] [PubMed]

67. Castro-Nallar, E.; Bendall, M.L.; Pérez-Losada, M.; Sabuncyan, S.; Severance, E.G.; Dickerson, F.B.; Schroeder, J.R.; Yolken, R.H.; Crandall, K.A. Composition, taxonomy and functional diversity of the oropharynx microbiome in individuals with schizophrenia and controls. PeerJ 2015, 3, e1140. [CrossRef]

68. Palleja, A.; Mikkelsen, K.H.; Forslund, S.K.; Kashani, A.; Allin, K.H.; Nielsen, T.; Hansen, T.H.; Liang, S.; Feng, Q.; Zhang, C.; et al. Recovery of gut microbiota of healthy adults following antibiotic exposure. Nat. Microbiol. 2018, 3, 1255-1265. [CrossRef]

69. Bahr, S.M.; Tyler, B.C.; Wooldridge, N.; Butcher, B.D.; Burns, T.L.; Teesch, L.M.; Oltman, C.L.; Azcarate-Peril, M.A.; Kirby, J.R.; Calarge, C.A. Use of the second-generation antipsychotic, risperidone, and secondary weight gain are associated with an altered gut microbiota in children. Transl. Psychiatry 2015, 5, e652. [CrossRef] 
70. Marlicz, W.; Yung, D.E.; Skonieczna-Żydecka, K.; Loniewski, I.; Van Hemert, S.; Loniewska, B.; Koulaouzidis, A. From clinical uncertainties to precision medicine: The emerging role of the gut barrier and microbiome in small bowel functional diseases. Expert Rev. Gastroenterol. Hepatol. 2017, 11, 961-978. [CrossRef]

71. Skonieczna-Żydecka, K.; Łoniewski, I.; Misera, A.; Stachowska, E.; Maciejewska, D.; Marlicz, W.; Galling, B. Second-generation antipsychotics and metabolism alterations: A systematic review of the role of the gut microbiome. Psychopharmacology 2018, 236, 1491-1512. [CrossRef]

72. Rao, T.S.S.; Asha, M.R.; Ramesh, B.N.; Rao, K.S.J. Understanding nutrition, depression and mental illnesses. Indian J. Psychiatry 2008, 50, 77-82.

73. Wu, G.D.; Chen, J.; Hoffmann, C.; Bittinger, K.; Chen, Y.-Y.; Keilbaugh, S.A.; Bewtra, M.; Knights, D.; Walters, W.A.; Knight, R.; et al. Linking long-term dietary patterns with gut microbial enterotypes. Science 2011, 334, 105-108. [CrossRef] [PubMed]

(C) 2019 by the authors. Licensee MDPI, Basel, Switzerland. This article is an open access article distributed under the terms and conditions of the Creative Commons Attribution (CC BY) license (http://creativecommons.org/licenses/by/4.0/). 